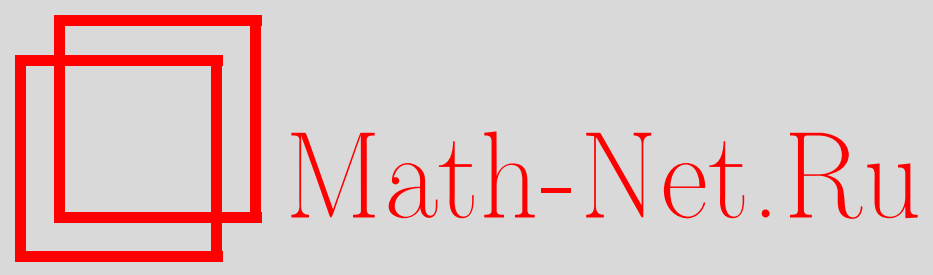

А. В. Болсинов, А. В. Борисов, Согласованные скобки Пуассона на алгебрах Ли, Матем. заметки, 2002, том 72, выпуск $1,11-34$

DOI: https://doi.org/10.4213/mzm400

Использование Общероссийского математического портала Math-Net.Ru подразумевает, что вы прочитали и согласны с пользовательским соглашением http://www.mathnet.ru/rus/agreement

Параметры загрузки:

IP : 3.85 .73 .92

26 апреля 2023 г., $07: 22: 39$

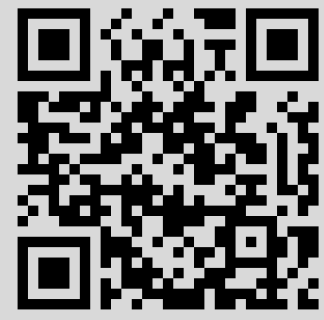




\title{
СОГЛАСОВАННЫЕ СКОБКИ ПУАССОНА НА АЛГЕБРАХ ЛИ
}

\author{
А.В. Болсинов, А. В. Борисов
}

\begin{abstract}
В работе обсуждается взаимосвязь между представлением интегрируемой системы в виде $L$ - $A$-пары со спектральным параметром и наличием у системы двух согласованных гамильтоновых представлений. Рассмотрены примеры согласованных скобок Пуассона на алгебрах Ли, отвечающие им интегрируемые гамильтоновы системы и представления Лакса.

Библиография: 30 названий.
\end{abstract}

1.1. Введение. Согласованные скобки Пуассона и гамильтоновы системы. Многие вполне интегрируемые гамильтоновы системы, возникающие в механике, математической физике и геометрии, обладают следующим замечательным свойством бигамильтоновости: они являются гамильтоновыми относительно сразу двух различных пуассоновых структур (см., например, [1]-[10]). Очень часто эти структуры оказываются согласованными между собой, а рассматриваемая система гамильтонова относительно любой их линейной комбинации (с постоянными коэффициентами).

С другой стороны, большинство интегрируемых гамильтоновых систем допускают запись в форме Лакса

$$
\dot{L}=[L, A]
$$

где $L, A$ - квадратные матрицы от фазовых переменных (говорят, что они образуют $L-A$-napy). Для интегрируемых систем типична возможность введения в элементы $L$ и $A$ произвольного (спектрального) параметра, которьй не входит в уравнения движения.

В этой работе исследуется взаимосвязь между представлением интегрируемой системы в виде $L$ - $A$-пары со спектральным параметром и наличием у системы двух согласованных гамильтоновых представлений.

Эта взаимосвязь, в некотором смысле, дает геометрическое и механическое обоснование формальньг алгебраических конструкций метода $L$ - $A$-пары, так как пуассонова структура (как и первые интегралы) является тензорньп инвариантом уравнений движения и имеет смысл (тензорного) закона сохранения. Отметим, что другие обоснования возможности введения спектрального параметра в $L$ - $A$-пару, например, метод $R$-матрицы [11] также имеют лишш формальный алгебраический смысл.

Работа выполнена при поддержке гранта Президента РФ, № 00-15-99272, и Российского фонда фундаментальных исследований, гранты № 99-01-01249 и № 98-01-00240. 
Напомним необходимые определения. Пусть $A=\left(A^{i j}\right)$ - кососимметрическое тензорное поле типа $(2,0)$ на многообразии $M$. Оно задает естественную операцию на пространстве гладких функций:

$$
\{f, g\}=A^{i j} \frac{\partial f}{\partial x^{i}} \frac{\partial g}{\partial x^{j}} .
$$

ОПРЕДЕЛЕНИЕ 1. Пуассоновой структурой на гладком многообразии $M$ назьвается кососимметричное тензорное поле $A^{i j}$ типа $(2,0)$ такое, что задаваемая им операция $\{$,$\} удовлетворяет тождеству Якоби:$

$$
\{f,\{g, h\}\}+\{f,\{g, h\}\}+\{f,\{g, h\}\}=0 \quad \text { для любьх } f, g, h \in C^{\infty}(M) .
$$

В этой ситуации пространство гладких функций получает естественную структуру бесконечномерной алгебры Ли, а операция $\{$,$\} назьвается скобкой Пуассона.$

ОПРЕДЕЛЕниЕ 2 . Пусть $f$ - гладкая функция. Векторное поле вида

$$
(\operatorname{sgrad} f)^{j}=A^{i j} \frac{\partial f}{\partial x^{i}}
$$

называется гамильтоновым. Функция $f$ при этом называется гамильтонианом. Инвариантное определение: $\operatorname{sgrad} f$ - это такое векторное поле, что для любой гладкой функции $g$ имеет место тождество

$$
\operatorname{sgrad} f(g)=\{f, g\}
$$

ОПРеДЕЛЕНИЕ 3. Две пуассоновы структуры $A$ и $B$ называются согласованными, если их сумма (или, что эквивалентно, произвольная линейная комбинация с постоянными коэффициентами) тоже является пуассоновой структурой (аналогично для скобок Пуассона).

КомМЕНТАРИЙ. Нетривиальное условие согласованности заключается в том, что сумма двух скобок Пуассона тоже удовлетворяет тождеству Якоби. Это условие можно переписать в аналитической форме: оно эквивалентно тому, что так назьваемая скобка Схоутена $\{\{A, B\}\}$ пуассоновых структур $A$ и $B$ равна нулю (см. [3], [12]). Кстати, само тождество Якоби для пуассоновой структуры $A$ записьвается в этих терминах в виде $\{\{A, A\}\}=0$.

ПримеР 1. Любые две постоянные скобки Пуассона согласованы.

Каждой скобке Пуассона можно естественньм образом сопоставить ее ранг. Это просто ранг матрищы $A^{i j}$ в точке общего положения. Ниже мы будем рассматривать только вещественно-аналитические скобки Пуассона, поэтому здесь мы всегда предполагаем, что точки общего положения образуют открытое всюду плотное подмножество в $M$.

Если $\operatorname{rank} A=\operatorname{dim} M$, то скобка Пуассона назьвается невырожсденной (почти всюду). Если она невырождена всюду, то можно рассмотреть обратньй тензор $A_{i j}$ или соответствующую ему дифференциальную 2-форму $\omega=A_{i j} d x^{i} \wedge d x^{j}$. Эта форма, как нетрудно проверить, является симплектической структурой, т.е. невырождена и замкнута. 
ОПРЕДЕЛЕНИЕ 4. Функция $f$ называется функиией Казимира пуассоновой структуры $A$, если

$$
\{f, g\} \equiv 0
$$

для любой гладкой функции $g$.

Если пуассонова структура вырождена, то локально в окрестности точки общего положения функции Казимира всегда существуют, причем число функционально независимых функций Казимира в точности равно корангу пуассоновой структуры corank $A=$ $\operatorname{dim} M-\operatorname{rank} A$.

ПРИмеР 2. Одним из важнейших примеров скобок Пуассона являются линейные скобки Пуассона, или скобки Пуассона-Ли. Линейность означает, что коэффициенты тензороного поля $A_{i j}(x)$ являются линейньми функциями от координат $x_{1}, \ldots, x_{n}$ (верхние и нижние индексы здесь удобно поменять местами). Легко видеть, что такие скобки находятся в естественном взаимно-однозначном соответствии с алгебрами Ли. Действительно, пусть $G$ - конечномерная алгебра Ли, $G^{*}-$ двойственное пространство. Определим на $G^{*}$ скобку Пуассона по формуле

$$
\{f, g\}(x)=x([d f(x), d g(x)]), \quad x \in G^{*}, \quad d f(x), d g(x) \in\left(G^{*}\right)^{*}=G .
$$

Эквивалентным образом в координатах эта скобка записьвается так:

$$
\{f, g\}(x)=c_{j k}^{i} x_{i} \frac{\partial f}{\partial x_{j}} \frac{\partial g}{\partial x_{k}},
$$

где $c_{j k}^{i}$ - компоненты структурного тензора алгебры $G$ в базисе, отвечающем координатам $x_{1}, \ldots, x_{n}$.

Обратно, если мы имеем линейную скобку Пуассона, т.е. $A_{j k}(x)=c_{j k}^{i} x_{i}$, то $c_{j k}^{i}-$ структурньй тензор некоторой алгебры Ли.

Функции Казимира скобки Пуассона-Ли - это в точности инварианты коприсоединенного представления соответствующей группы Ли $\mathfrak{G}$ на $G^{*}$.

Рассмотрим теперь следующую ситуацию: векторное поле является гамильтоновым относительно двух согласованных между собой пуассоновых структур. Такие векторные поля называются бигамильтоновыми. Известно, что бигамильтоновы векторные поля обычно обладают большим запасом первьх интегралов, которые могут быть построены при помощи двух конструкций, обсуждаемых ниже по отдельности в вырожденном и невырожденном случае (см., например, [1], [3], [7], [9], [13]).

1.2. Случай невырожденных пуассоновых структур. Пусть даны две согласованные пуассоновы структуры $A_{0}, A_{1}$, одна из которых, скажем $A_{0}$, невырождена.

Принципиальное отличие такой ситуации от вырожденного случая состоит в том, что мы можем корректно определить так назьваемьй оператор рекурсии $P=A_{1} A_{0}^{-1}$, играюший важную роль в дальнейшей конструкции и интересньй сам по себе. Если рассматривать $A_{i}$ как оператор из кокасательного пространства $T_{x}^{*} M$ в касательное $T_{x} M$, то $P$ - это линейньй оператор в касательном пространстве, т.е. $P: T_{x} M \rightarrow T_{x} M$. Вместе с ним мы будем рассматривать сопряженньй оператор $P^{*}: T_{x}^{*} M \rightarrow T_{x}^{*} M$, который, очевидно, задается формулой $P^{*}=A_{0}^{-1} A_{1}$.

Следующее утверждение показывает, как условие согласованности $A_{0}$ и $A_{1}$ может быть переформулировано в терминах оператора рекурсии. 
ПРЕДЛОЖЕНИЕ 1. Пусть $A_{0} u A_{1}$ - пуассоновы структуры, причем $A_{0}$ невырождена. Тогда следующие условия әквивалентны:

1) пуассоновы структуры $A_{0}$ и $A_{1}$ согласованы;

2) дифференциальная 2-форма $\Omega_{1}=A_{0}^{-1} P$, задаваемая тождеством $\Omega_{1}(\xi, \eta)=$ $A_{0}^{-1}(P \xi, \eta)$, замкнута;

3) тензор Нийенхейса, отвечающий оператору рекурсии $P$, тождественно равен нулю.

Мы не будем приводить доказательства этого утверждение, ограничившись ссылкой, например, на [5], [6], [14]. Напомним лишш, что тензор Нийенхейса, отвечающий произвольному оператору $P$, определяется как билинейная векторнозначная форма на парах векторных полей $u, v$, задаваемая следующей формулой:

$$
N_{P}(u, v)=-P^{2}[u, v]+P[P u, v]+P[u, P v]-[P u, P v] .
$$

Следующее утверждение показывает, что пара согласованных пуассоновых структур $A_{0}, A_{1}$ позволяет построить целое семейство попарно согласованных структур $A_{k}$ $(k \in \mathbb{N})$.

ПРЕДЛОЖЕНИЕ 2. Бивекторное поле вида $A_{k+1}=A_{1}\left(P^{*}\right)^{k}$ является пуассоновой структурой для любого $k \in \mathbb{N}$, причем все такие структуры попарно согласованы межуду собой, а также с $A_{0} u A_{1}$.

ДокАзАтЕльство. См., например, [13]. Поясним, что выражение $A_{1}\left(P^{*}\right)^{k}$ обозначает билинейную форму на кокасательном пространстве, определенную по естественной формуле $A_{k+1}(a, b)=A_{1}\left(P^{* k} a, b\right)$. Косая симметрия этой формы сразу следует из определения оператора рекурсии, т.е. $A_{k+1}$ действительно является бивектором.

ЗАмЕчАниЕ. В описанной вьше ситуации говорят, что пуассоновы структуры $A_{k}$ задают иерархию пуассоновых структур. Следует, впрочем, заметить, что все они получаются из исходных структур $A_{0}$ и $A_{1}$ при помоши стандартных тензорных операций и поэтому в естественном смысле не являются независимыми. Кроме того, они удовлетворяют системе линейных соотношений, коэффициентами которых являются коэффициенты характеристического многочлена оператора рекурсии. Действительно, если $Q(t)=t^{n}+a_{n-1} t^{n-1}+\cdots+a_{1} t+a_{0}-$ характеристический многочлен для $P$ (или, что то же самое, для $P^{*}$ ), то по теореме Гамильтона-Кэли

$$
P^{* n}+a_{n-1} P^{* n-1}+\cdots+a_{1} P^{*}+a_{0} E=0 .
$$

Поэтому для любого $m>n$ имеем

$$
A_{m}+a_{n-1} A_{m-1}+\cdots+a_{1} A_{m-n+1}+a_{0} A_{m-n}=0
$$

Рассмотрим теперь бигамильтоново векторное поле

$$
v=\operatorname{sgrad}_{0} f_{1}=\operatorname{sgrad}_{1} f_{0} \text {. }
$$

Конструкция, которая позволяет построить набор его первых интегралов и обычно приводит к полной интегрируемости, состоит в следующем. 
Рассмотрим векторное поле вида $\operatorname{sgrad}_{1} f_{1}$. Оказьвается, оно снова является бигамильтоновым, т.е. существует функция $f_{2}$ такая, что

$$
\operatorname{sgrad}_{1} f_{1}=\operatorname{sgrad}_{0} f_{2} .
$$

Продолжая процесс (1) по индукции, мы получаем систему рекуррентных соотношений

$$
\operatorname{sgrad}_{1} f_{k}=\operatorname{sgrad}_{0} f_{k+1} .
$$

Утверждается, что все функции $f_{k}$ являются первыми интегралами бигамильтонова поля $v$, коммутирующими между собой.

Легко видеть, что в терминах оператора рекурсии эта система соотношений может быть записана в следующем естественном виде: $d f_{k+1}=P^{* k+1} d f_{0}$ (т.е. $\left.d f_{k+1}=P^{*} d f_{k}\right)$, или в терминах гамильтоновых векторных полей: $\operatorname{sgrad}_{1} f_{k}=P^{k} v$.

ПРЕДЛОЖЕНИЕ 3. 1) Векторные поля вида $P^{k} v$ являются гамильтоновы.ми относительно пуассоновых структур $A_{0} u A_{1}$. Другими словами, существуют гладкие функиии $f_{k}$ такие, что $\operatorname{sgrad}_{1} f_{k}=P^{k} v$. Эти функиии удовлетворяют системе рекуррентных соотношений

$$
\operatorname{sgrad}_{1} f_{k}=\operatorname{sgrad}_{0} f_{k+1} .
$$

2) Функиии $f_{k}$ попарно коммутируют относительно скобок Пуассона $\{,\}_{0} u$ $\{,\}_{1}$. В частности, все они являются первыми интегралами бигамильтонова поля $v$.

3) Если $A_{1}$ невьрожсдена, то векторные поля $P^{m} v$ являются гамильтоновыми относительно каждой из пуассоновых структур $A_{k}=P^{k} A_{0}$. Функции $f_{m}$ попарно коммутируют относительно каждой из соответствующих скобок $\{,\}_{k}$.

ЗАмЕчАниЕ. Обе пуассоновы структуры $A_{0}$ и $A_{1}$ являются тензорными инвариантами бигамильтонова векторного поля $v$. Отсюда сразу следует, что инвариантным является характеристический многочлен $\operatorname{det}\left(A_{1}-\lambda A_{0}\right)$. В частности, его корни (т.е. собственные числа оператора рекурсии), рассматриваемые как функции на многообразии, являются первыми интегралами векторного поля $v$.

Возникает естественньй вопрос: какие гамильтонианы дают бигамильтоновы векторные поля для данной пары согласованньх пуассоновых структур $A_{0}$ и $A_{1}$ ? Для разных типов $A_{0}$ и $A_{1}$ локальньй ответ был получен П. Олвером в [12]. Мы ограничимся здесь двумя простейшими, но важньми примерами.

ПримеР 3. Пусть собственные значения оператора рекурсии вещественны, различны и постоянны. Тогда (локально) существует система координат $p_{1}, \ldots, p_{n}, q_{1}, \ldots, q_{n}$, в которой

$$
\begin{aligned}
& \left\{p_{i}, p_{j}\right\}_{0}=\left\{q_{i}, q_{j}\right\}_{0}=0, \quad\left\{p_{i}, q_{j}\right\}_{0}=\delta_{i j}, \\
& \left\{p_{i}, p_{j}\right\}_{1}=\left\{q_{i}, q_{j}\right\}_{1}=0, \quad\left\{p_{i}, q_{j}\right\}_{1}=a_{i} \delta_{i j},
\end{aligned}
$$

где $a_{1}, \ldots, a_{n}$ - собственные значения оператора рекурсии. Тогда несложно проверить, что бигамильтоновы системы задаются в точности гамильтонианами с разделенными переменными:

$$
H=F_{1}\left(p_{1}, q_{1}\right)+F_{2}\left(p_{2}, q_{2}\right)+\cdots+F_{n}\left(p_{n}, q_{n}\right) .
$$

Условие, при котором иерархия (1) порождает полньй набор первых интегралов, может быть записано в следующей инвариантной форме. Пусть $L_{i}$ - двумерные собственные подпространства оператора рекурсии $P$. Тогда $d H\left(L_{i}\right) \neq 0$ для любого $i=1, \ldots, n$. 
ПРИМеР 4. Пусть собственные значения оператора рекурсии различны и все непостоянны. Оказывается, в этом случае они автоматически будут функционально независимыми попарно коммутирующими функциями. В частности, отсюда будет следовать полная интегрируемость любой бигамильтоновой системы. Отметим также, что в качестве гамильтонианов, дающих нетривиальные бигамильтоновы системы, можно рассмотреть функции вида

$$
H_{k}(x)=\operatorname{Tr} P^{k}(x) .
$$

В их бигамильтоновости легко убедиться, воспользовавшись равенством нулю тензора Нийенхейса $N_{P}$, которое можно эквивалентным образом переписать в виде $P \mathscr{L}_{v} P-$ $\mathscr{L}_{P v} P=0$ (здесь $\mathscr{L}_{v}-$ производная Ли вдоль векторного поля $v$ ). Тогда для любого векторного поля $v$ мы имеем (см. [13])

$$
\begin{aligned}
d H_{k}(v) & =\mathscr{L}_{v} \operatorname{Tr} P^{k}=\operatorname{Tr} \mathscr{L}_{v} P^{k}=\operatorname{Tr} k P^{k-1} \mathscr{L}_{v} P=\operatorname{Tr} k P^{k-2} \mathscr{L}_{P v} P \\
& =\frac{k}{k-1} \operatorname{Tr} \mathscr{L}_{P v} P^{k-1}=\frac{k}{k-1} \mathscr{L}_{P v} \operatorname{Tr} P^{k-1} \\
& =\frac{k}{k-1} d H_{k-1}(P v)=\frac{k}{k-1} P^{*} d H_{k-1}(v) .
\end{aligned}
$$

Таким образом,

$$
d H_{k}=\frac{k}{k-1} P^{*} d H_{k-1},
$$

что и означает бигамильтоновость. Отметим, что функции вида $\operatorname{Tr} P^{k}$ всегда дают бигамильтоновы системы. Однако для того, чтобы они давали полньй набор интегралов в инволюции, необходима простота спектра и непостоянство собственных значений. При выполнении этих условий любая бигамильтонова система является вполне интегрируемой.

Более подробно локальная структура невырожденной пары согласованных пуассоновых структур описана в [12], [13].

1.3. Случай вырожденных скобок Пуассона. Рассмотрим теперь линейное семейство вырожденных пуассоновых структур $J=\left\{\lambda_{0} A_{0}+\lambda_{1} A_{1}\right\}$ на многообразии $M$ (такая ситуация подробно изучается в [1], [9], [15]. Для каждой структуры $C \in J$ мы можем определить ее ранг $\operatorname{rank} C$.

Поскольку мы предположили, что все рассматриваемые скобки являются вырожденными, то $\operatorname{rank} C<\operatorname{dim} M$. Пусть $R_{0}=\max _{C \in J} \operatorname{rank} C$. Ясно, что почти все скобки из рассматриваемого семейства имеют ранг $R_{0}$. Мы будем называть их скобками общего положения. Исключением может быть лиш конечное число скобок (с точностью до пропорциональности). Подсемейство скобок общего положения мы обозначим через $J_{0}$.

Пусть $\mathscr{Z}(C)$ - множество функций Казимира пуассоновой структуры $C \in J$. Следующее утверждение дает рецепт построения большого набора функций, находящихся в инволюции относительно всех скобок из рассматриваемого семейства.

ПредЛОЖЕНИЕ 4. 1) Рассмотрим две произвольные скобки $B=\lambda_{0} A_{0}+\lambda_{1} A_{1} u$ $B^{\prime}=\mu_{0} A_{0}+\mu_{1} A_{1}$ из семейства $J$. Пусть они непропорииональны, т.е. $\lambda_{0} \mu_{1}-$ $\lambda_{1} \mu_{0} \neq 0$. Тогда функиии Казимира этих скобок $f \in \mathscr{Z}(B), g \in \mathscr{Z}\left(B^{\prime}\right)$ находятся в инволюиии относительно любой скобки $C \in J$.

2) Множество функиий Казимира $\mathscr{Z}(B)$ является алгеброй Ли относительно любой скобки $C \in J$. Если $B \in J_{0} \subset J$ - скобка общего положсения (т.е. $\left.\operatorname{rank} B=R_{0}\right)$, то алгебра Ли $\mathscr{Z}(B)$ коммутативна. 
ДокАЗАТЕЛЬСТВО. 1) Поскольку скобки $B$ и $B^{\prime}$ непропорциональны, то любую скобку $C \in J$ можно представить в виде линейной комбинации $\mathrm{C}=a B+a^{\prime} B^{\prime}$. Тогда

$$
\{f, g\}_{C}=a\{f, g\}_{B}+a^{\prime}\{f, g\}_{B^{\prime}}=0,
$$

так как $f \in \operatorname{ker}\{,\}_{B}=\mathscr{Z}(B), g \in \operatorname{ker}\{,\}_{B^{\prime}}=\mathscr{Z}\left(B^{\prime}\right)$.

2) Тот факт, что $\mathscr{Z}(B)$ является подалгеброй, легко проверяется непосредственной проверкой. Второе утверждение получается, например, переходом к пределу в доказанном только что равенстве при $B^{\prime} \rightarrow B$. Отметим, что условие "общности положения" для $B$ является существенным. Если оно не выполняется, то, переходя к пределу, мы, грубо говоря, получим не все функции Казимира скобки $B$, а лишь некоторое подпространство.

Таким образом, используя пару согласованных вырожденных скобок Пуассона, мы можем построить достаточно большое семейство функций в инволюции $\mathscr{F}_{J_{0}}$, объединив функции Казимира всех скобок общего положения:

$$
\mathscr{F}_{J_{0}}=\bigcup_{B \in J_{0}} \mathscr{Z}(B) .
$$

Предположим теперь, что мы имеем динамическую систему, которая одновременно является гамильтоновой относительно всех (нетривиальных) скобок из рассматриваемого семейства. Ясно, что все функции из семейства $\mathscr{F}_{J_{0}}$ будут ее первыми интегралами. В каком случае этих интегралов хватает для полной интегрируемости? Другими словами, когда число функционально независимых функций в семействе равно

$$
\frac{1}{2} \operatorname{rank} C+\operatorname{corank} C=\operatorname{dim} M-\frac{1}{2} R_{0},
$$

где $C \in J_{0}$ - скобка общего положения? Это условие на самом деле означает, что подпространство в $T_{x}^{*} M$, порожденное дифференциалами функций $g \in \mathscr{F}_{J_{0}}$, является максимальньм изотропным подпространством относительно формы $C$ (почти всюду на $M$ ).

Имеется эффективный критерий [1], позволяющий проверять полноту семейства $\mathscr{F} J_{0}$. Более того, в случае, когда семейство является полньп, этот критерий указьвает, в каких точках многообразия первые интегралы становятся функционально зависимыми.

Для произвольного комплексного числа $\lambda \in \mathbb{C}$ определим подмножество "сингулярных точек" в $M$ :

$$
S_{\lambda}=\left\{x \in M \mid \operatorname{rank}\left(A_{0}(x)+\lambda A_{1}(x)\right)<R_{0}\right\} .
$$

Кроме того формально положим $S_{\infty}=\left\{x \in M \mid \operatorname{rank} A_{1}(x)<R_{0}\right\}$.

Предположим теперь, что все скобки из рассматриваемого семейства обладают глобально определенными функциями Казимира, причем эти функции локально разделяют симплектические листы максимальной размерности. Другими словами, если $\operatorname{rank} B(x)=R_{0}$, то подпространство $\operatorname{ker} B(x)$ порождается дифференциалами $d f(x)$ функций Казимира $f \in \mathscr{Z}(B)$.

Tеорема 1 [1]. Пусть $L_{x} \subset T_{x}^{*} M-$ подпространство, порохсденое дифференииалами функиий $g \in \mathscr{F}_{J_{0}}$. Тогда следующие условия әквивалентны:

1) $L_{x}$ - максимальное изотропное подпространство относительно формы $C(x), C \in J_{0} ;$

2) $x \notin S=\bigcup_{\lambda \in \overline{\mathbb{C}}} S_{\lambda}$. 
Таким образом, семейство $\mathscr{F}_{J_{0}}$ является полным тогда и только тогда, когда дополнение к множеству $S$ всюду плотно в $M$. В частности, необходимым условием полноты является то, что все скобки в семействе $J$ имеют одинаковьй ранг $R_{0}$. Действительно,

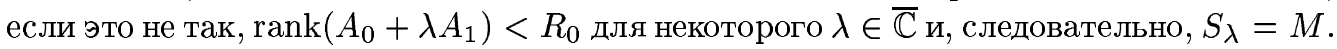
Однако достаточным это условие не является. Грубо говоря, дополнительно требуется, чтобы сингулярные множества $S_{\lambda}$ для каждой скобки $\{,\}_{\lambda}$ были не слишком велики.

Следующая конструкция дает возможность строить примеры бигамильтоновых систем для данного семейства вырожденных согласованных скобок Пуассона $\lambda_{0} A_{0}+\lambda_{1} A_{1}$. Оказывается, в качестве соответствующих гамильтонианов можно брать функции Казимира скобок общего положения.

Будем считать без ограничения общности, что $A_{0}$ - скобка общего положения, и рассмотрим функции Казимира линейной комбинации $A_{0}-\lambda A_{1}$ как функции, зависящие от параметра $\lambda$. Предположим (а именно так и происходит в реальных задачах), что зависимость от параметра $\lambda$ является аналитической в окрестности нуля (т.е. скобки $\left.A_{0}\right)$ :

$$
f_{\lambda}(x)=f_{0}(x)+\lambda f_{1}(x)+\lambda^{2} f_{2}(x)+\cdots \in \mathscr{Z}\left(A_{0}+\lambda A_{1}\right) .
$$

В частности, $f_{0}$ - функция Казимира скобки $A_{0}$.

ПРЕДЛОЖЕНИЕ 5. При сделанных предположениях векторное поле $v=\operatorname{sgrad}_{1} f_{0}$ $=A_{1}\left(d f_{0}\right)$ является гамильтоновым относительно скобки $A_{0}$, более того, относительно любой линейной комбиначии $A_{0}-\lambda A_{1}$.

ДокАЗАТЕЛЬСТВо. Поскольку $f_{\lambda}$ является функцией Казимира для $A_{0}-\lambda A_{1}$, то

$$
\left(A_{0}-\lambda A_{1}\right)\left(d f_{0}+\lambda_{1} d f_{1}+\lambda^{2} d f_{2}+\cdots\right) \equiv 0 .
$$

Приравнивая члены при одинаковых степенях $\lambda$, мы получаем систему рекуррентных соотношений:

$$
A_{0}\left(d f_{0}\right)=0, \quad A_{0}\left(d f_{1}\right)=A_{1}\left(d f_{0}\right), \quad \ldots \quad A_{0}\left(d f_{k}\right)=A_{1}\left(d f_{k-1}\right), \ldots
$$

Второе соотношение означает, что векторное поле $v$ является гамильтоновьм относительно скобки $A_{0}$ с гамильтонианом $f_{1}$. Кроме того, очевидно, что $v$ является гамильтоновым относительно скобки $A_{0}-\lambda A_{1}$ с гамильтонианом $-f_{0} / \lambda$. Предложение доказано.

Итак, в случае вырожденных скобок Пуассона мы описали один из механизмов появления бигамильтоновых систем и указали достаточные условия для проверки их полной интегрируемости. Ниже мы покажем, как эта конструкция проявляется в конкретных примерах из механики. Подчеркнем, однако, что условия полноты семейства $\mathscr{F}_{J_{0}}$ не следует рассматривать как необходимые условия интегрируемости. Более того, имеется ряд способов, позволяющих дополнить семейство $\mathscr{F} J_{0}$ в тех случаях, когда оно полным не является.

\section{2. Представление Лакса с параметром и полупростые скобки Пуассона.}

ОПРЕДЕЛЕНИЕ 5. Дифференииальным уравнением в форме Лакса назьвают уравнение вида

$$
\frac{d L}{d t}=[L, A]
$$


где $L(x), A(x)$ - некоторые квадратные матрицы, зависящие от фазовых переменных $x$.

Это уравнение обладает некоторыми замечательными свойствами. Например, функции вида $\operatorname{Tr} L^{k}$ являются его первьми интегралами. Иногда это уравнение удается переписать в следующем эквивалентном виде:

$$
\frac{d L(\lambda)}{d t}=[L(\lambda), A(\lambda)]
$$

где $\lambda$ - некоторый параметр, который не входит в уравнения движения. В этом случае интегралов становится больше: ими будут функции вида $\operatorname{Tr} L(\lambda)$ для любого $\lambda \in \mathbb{R}$. Обьчно таких функций хватает для полной интегрируемости.

Хорошо известно, что если алгебра Ли $G$ является полупростой, то уравнения на $G^{*}$, являюшиеся гамильтоновыми относительно скобки Пуассона-Ли (см. пример 2), могут быть записаны в лаксовой форме. Действительно, отождествим пространства $G$ и $G^{*}$ при помощи скалярного произведения $(X, Y)=\operatorname{Tr} X Y$, где $X, Y$ - элементы $G$ в некотором матричном представлении. Тогда гамильтонова система на $G^{*}=G$ с гамильтонианом $H$ переписьвается в виде

$$
\frac{d X}{d t}=\operatorname{ad}_{d H(X)}^{*} X,
$$

где оператор коприсоединенного представления $\mathrm{ad}^{*}$ определяется тождеством

$$
\left\langle\operatorname{ad}_{d H(X)}^{*} Y, \xi\right\rangle=\langle Y,-[d H(X), \xi]\rangle
$$

Учитывая наш способ отождествления, получаем

$$
\operatorname{Tr}\left(\operatorname{ad}_{d H(X)}^{*} Y\right) \xi=-\operatorname{Tr} Y(d H(X) \xi-\xi d H(X))=\operatorname{Tr}(d H(X) Y-Y d H(X)) \xi .
$$

Отсюда $\operatorname{ad}_{d H(X)}^{*} Y=d H(X) Y-Y d H(X)=[d H(X), Y]$. Таким образом, в полупростом случае присоединенное и коприсоединенноепредставления совпадают и, следовательно, гамильтонова система (2) переписьвается в виде

$$
\frac{d X}{d t}=[d H(X), X] .
$$

Аналогичным образом, для бигамильтоновых систем справедливо следующее общее утверждение.

ТЕорема 2. Пусть $\{,\}_{\lambda}$ - семейство скобок Пуассона на некотором линейном пространстве. Пусть почти все эти скобки Пуассона являются (изоморфны) “полупростыми” скобками Ли-Пуассона. Предположим, что система $v$ является гамильтоновой относительно всех скобок из этого семейства, т.е. допускает представление в виде

$$
v(x)=\operatorname{sgrad}_{\lambda} H_{\lambda}(x),
$$

әде $H_{\lambda}$ - гамильтониан, отвечающий скобке $\{,\}_{\lambda}$. Тогда для $v$ существует представление Лакса с параметром $\lambda$ (который, впрочем, мохсет оказаться не раииональным, а более сложным). 
ДокАЗАТЕЛЬСТво этого факта довольно естественно. Если система $v$ гамильтонова относительно скобки Ли-Пуассона, отвечающей полупростой алгебре Ли, то, отождествляя двойственное пространство алгебры с самой алгеброй, мы получаем в точности представление Лакса для $v$ (пока без параметра). Поскольку в рассматриваемом случае мы имеем дело с семейством $\{,\}_{\lambda}$ полупростых скобок, то в результате отождествления (которое также зависит от $\lambda$ ) мы получим семейство представлений Лакса с параметром $\lambda$, что и требовалось.

КомменТАРИй. Отметим, что если рассматриваемое семейство содержит хотя бы одну “полупростую скобку", то почти все его скобки также являются полупростыми (при условии, что мы имеем дело с линейными скобками или сводящимися к ним). Более того, все пространство параметров разделяется на открытые камеры, каждая из которых "содержит" изоморфные между собой “полупростые скобки".

Теперь мы продемонстрируем описанную конструкцию на нескольких конкретных примерах.

3. Согласованные скобки Пуассона, связанные с методом сдвига аргумента. Примеры: геодезические левоинвариантных метрик на полупростых группах Ли и волчок на $s o(4)$. Пусть $G$-произвольная алгебра Ли, $G^{*}$ - двойственное пространство. Кроме стандартной скобки Пуассона-Ли $\{f, g\}(x)=x([d f(x), d g(x)])$ рассмотрим на $G^{*}$ постоянную скобку, которая получается из нее "замораживанием аргумента":

$$
\{f, g\}_{a}(x)=a([d f(x), d g(x)]), \quad \text { где } a \in G^{*} .
$$

Легко проверяется, что эти скобки согласованы. Поэтому в силу общей конструкции функции Казимира линейных комбинаций вида $\{\}+,\lambda\{$,$\} находятся в инволюции.$

Легко видеть, что такая линейная комбинация переходит в стандартную скобку при замене $x \rightarrow x+\lambda a$. В частности, ее функции Казимира имеют вид $f\left(x+\lambda_{a}\right)$, где $f$ пробегает кольцо инвариантов коприсоединенного представления $I(G)$. Таким образом, на коалгебре Ли мы получаем набор функций в инволюции $\{f(x+\lambda a)\} f \in I(G), \lambda \in \mathbb{R}$. Этот метод конструирования функций в инволюции был предложен А. С. Мищенко и А. Т. Фоменко [16], [17] как обобщение конструкции С. В. Манакова [18]. Ими же было показано, что такой набор функций является полным в случае полупростой алгебры Ли, и описан класс квадратичных гамильтонианов, для которых функции $f(x+\lambda a)$ являются первыми интегралами. В случае, когда $G$ полупроста, соответствующие системы в точности совпадают с системами, гамильтоновыми относительно любой линейной комбинации $\{\}+,\lambda\{,\}_{a}$ (в случае квадратичных гамильтонианов) (см. [4]).

Опишем соответствующую конструкцию более подробно. Пусть $G$ полупроста. Тогда мы можем отождествить ее с двойственным пространством $G^{*}$ при помощи метрики Киллинга и считать, что ковектор а является элементом самой алгебры $G$. Предположим, что $a$ является полупростым регулярным элементом, и рассмотрим порожденную им подалгебру Картана $K$ (которая в данном случае совпадает с его централизатором $K=\{x \in G \mid[x, a]=0\})$. Определим теперь самосопряженньй оператор $C: G \rightarrow G$ по следующему правилу. Представим произвольньй элемент $x \in G$ в виде суммы $x=x_{1}+x_{2}$, где $x_{1} \in K, x_{2} \in K^{\perp}$, и положим $C(x)=\operatorname{ad}_{a}^{-1} \operatorname{ad}_{b} x_{2}+D\left(x_{1}\right)$, где $\operatorname{ad}_{a}^{-1}: K^{\perp} \rightarrow K^{\perp}$ корректно определен, а $D: K \rightarrow K$ - произвольный самосопряженный оператор. 
ПРЕДЛОЖЕНИЕ 6 [16],[17]. Гамильтонова система

$$
\dot{x}=[C x, x]
$$

с гамильтонианом $h(x)=\frac{1}{2}(C x, x)$ на алгебре Ли $G$ является вполне интегрируемой. Ее первыми интегралами являются функиии вида $f(x+\lambda a)$, где $f$ - инвариант присоединенного представления, $\lambda \in \mathbb{R}$. Эта система является гамильтоновой относительно любой линейной комбиначии $\{\}+,\lambda\{,\}_{a} u$, следовательно, допускает представление Лакса со спектральным параметром

$$
\frac{d}{d t}(x+\lambda a)=[C(x)-b, x+\lambda a]
$$

Имеется важный частньй случай, когда система (4) может быть естественньм образом ограничена на некоторую подалгебру $H \subset G$ (другими словами, подалгебра $H$ является "инвариантным подмногообразием" данной системы). Примером такой подалгебры является, например, так называемая нормальная форма комплексной алгебры Ли $G^{\mathbb{C}}$ (см. [16], [17]). Простейшим случаем такой ситуации является волчок Манакова. Здесь $G=s l(n, \mathbb{R}), H=s o(n, \mathbb{R}) \subset G$. В качестве подалгебры Картана берется подалгебра диагональных матрищ. В частности, $a$ и $b$ - это диагональные матрицы $A=\operatorname{diag}\left(a_{1}, \ldots, a_{n}\right), B=\operatorname{diag}\left(b_{1}, \ldots, b_{n}\right)$. Тогда гамильтониан $h$, ограниченный на подалгебру $H$, имеет вид $h=\frac{1}{2}\left(a d_{a}^{-1} \operatorname{ad}_{b} X, X\right), X \in s o(n)$. Если положить $A=B^{2}=\operatorname{diag}\left(b_{1}^{2}, \ldots, b_{n}^{2}\right)$, то $h(X)=\sum\left(b_{i}+b_{j}\right)^{-1} x_{i j}$, и, подставляя это выражение в (5), мы получаем представление Лакса со спектральным параметром, указанное Манаковым в [18]:

$$
\frac{d}{d t}\left(X+\lambda B^{2}\right)=\left[\Omega-B, X+\lambda B^{2}\right]
$$

где $\Omega(X)=\operatorname{dh}(X)$ и $X$ связаны соотношением $X=B \Omega+\Omega B$.

Мищенко и Фоменко в [16], [17] показали, что такая конструкция работает в случае произвольных нормальных форм, и описали соответствующие интегрируемые системы, которые они интерпретировали как геодезические потоки левоинвариантных метрик на групшах Ли. В частности, ими была указана интересная система на алгебре Ли $s o(4)$, которую можно построить, повторяя изложенную выше конструкцию для пары $H=s o(4), G=g_{2}$. В результате на $s o(4)$ возникает новое семейство квадратичных гамильтонианов с дополнительньм интегралом степени 4. Позднее этот интегрируемый случай был переоткрыт в работах М. Адлера, П. ван Мербеке [19] и А. Г. Реймана, М. А. Семенова-Тян-Шанского [20].

Явный вид соответствующей гамильтоновой системы таков. Рассмотрим реализацию алгебры Ли $g_{2}$ в виде подалгебры алгебры Ли $s o(4,3)$, образованную матрицами вида

$$
\left(\begin{array}{ccccccc}
0 & -\frac{u_{3}+w_{3}}{2} & \frac{u_{2}+w_{2}}{2} & -\frac{u_{1}-w_{1}}{2} & -y_{2} & y_{3} & a_{1} \\
\frac{u_{3}+w_{3}}{2} & 0 & -\frac{u_{1}+w_{1}}{2} & -\frac{u_{2}-w_{2}}{2} & y_{1} & a_{2} & z_{3} \\
-\frac{u_{2}+w_{2}}{2} & \frac{u_{1}+w_{1}}{2} & 0 & -\frac{u_{3}-w_{3}}{2} & a_{3} & z_{1} & -z_{2} \\
\frac{u_{1}-w_{1}}{2} & \frac{u_{2}-w_{2}}{2} & \frac{u_{3}-w_{3}}{2} & 0 & y_{3}-z_{3} & y_{2}-z_{2} & y_{1}-z_{1} \\
-y_{2} & y_{1} & a_{3} & y_{3}-z_{3} & 0 & w_{1} & -w_{2} \\
y_{3} & a_{2} & z_{1} & y_{2}-z_{2} & -w_{1} & 0 & w_{3} \\
a_{1} & z_{3} & -z_{2} & y_{1}-z_{1} & w_{2} & -w_{3} & 0
\end{array}\right)
$$


где $a_{1}+a_{2}+a_{3}=0$. Подалгебра $s o(4) \subset g_{2}$ является максимальной компактной подалгеброй и состоит из матриц, отвечающих переменным $u_{i}, w_{i}$. В качестве гамильтониана на $s o(4)=s o(4) *$ рассматривается квадратичная форма вида

$$
H(X)=\operatorname{Tr}\left(\operatorname{ad}_{a}^{-1} \operatorname{ad}_{b} X\right) X
$$

где $a$ и $b$ - элементы подалгебры Картана $K$, состоящей из матрищ с произвольными элементами на побочной диагонали и остальными нулями (при этом элемент $a$ берется регулярньм), т.е.

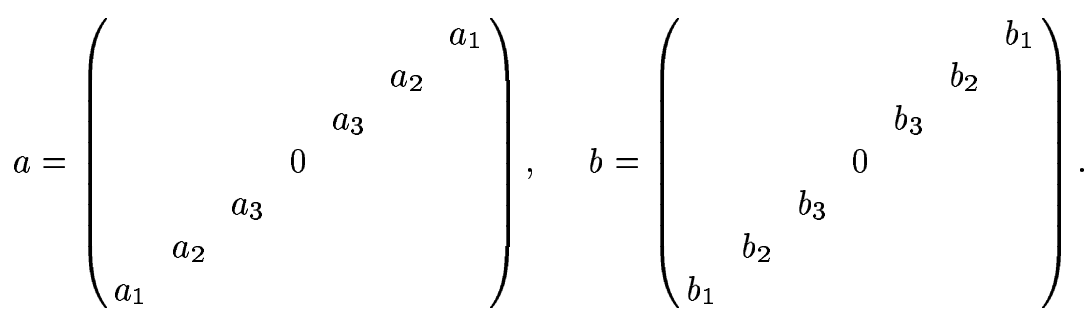

Как и в общем случае, представление Лакса имеет вид (5), где $x \in s o(4) \subset g_{2}$, а первые интегралы имеют вид $\operatorname{Tr}(X+\lambda a)^{k}$. Кроме гамильтониана и стандартных функций Казимира это дает еще один дополнительньй интеграл четвертой степени:

$$
I_{4}=\operatorname{Tr}\left(2 X^{4} a^{2}+2 X^{3} a X a+X^{2} a X^{2} a\right)
$$

После исключения функций Казимира гамильтониан и интеграл могут быть представлены в следующем явном виде:

$$
\begin{aligned}
H= & -\frac{2}{3} \sum_{k} a_{i}^{2} a_{j}^{2} u_{k}^{2}+\frac{2}{3} \sum_{k}\left(a_{k}^{4}-a_{i} a_{j}\left(a_{i}^{2}+a_{j}^{2}+\frac{5}{4} a_{i} a_{j}\right)\right) w_{k}^{2} \\
& +\sum_{k}\left(a_{k}^{4}+a_{i}^{2} a_{j}^{2}-\left(a_{i}+a_{j}\right)^{2}\left(a_{i}^{2}+a_{j}^{2}\right)\right) u_{k} w_{k}, \\
F= & \frac{1}{2} u^{2} \sum_{k}\left(\left(a_{i} a_{j}-\frac{1}{3} a_{k}^{2}\right) w_{k}^{2}+\left(a_{i} a_{j}-a_{k}^{2}\right) u_{k} w_{k}\right) \\
& +\frac{1}{18} w^{2} \sum_{k}\left(\frac{5}{3}\left(a_{i} a_{j}-a_{k}^{2}\right) w_{k}^{2}+\left(7 a_{i} a_{j}-4 a_{k}^{2}\right) u_{k} w_{k}\right) \\
& +\frac{1}{2} a^{2}\left(u^{2}+\frac{1}{3} w^{2}\right)(u, w)-\frac{1}{9} \sum_{i<j}\left(a_{i}-a_{j}\right)^{2}\left(u_{i} w_{i} w_{j}^{2}+u_{j} w_{j} w_{i}^{2}\right) .
\end{aligned}
$$

Здесь $u^{2}=\sum_{k} u_{k}^{2}, w^{2}=\sum_{k} w_{k}^{2}, a^{2}=\sum_{k} a_{k}^{2},(u, w)=\sum_{k} u_{k} w_{k}$.

Точно таким же способом интегрируемые системы могут быть построены на других аналогичных парах $H \subset G$, где $H$ - нормальная подалгебра в $G$. Например, можно построить интегрируемьй волчок на алгебре Ли $s o(16)$, используя вложение $s o(16) \subset e(8)$. Интегралы, полученные таким способом, будут иметь степени до 28 включительно, тогда как для волчка Манакова наибольшая степень интеграла - 14. 
4. Система Жуковского-Вольтерра. Описанная вьше конструкция "сдвига аргумента" допускает следующую модификацию в случае алгебры Ли $s o(3)$.

Рассмотрим на алгебре Ли so(3) новую скобку Пуассона-Ли $\{,\}_{B}$, соответствующую нестандартному коммутатору $[,]_{B}$, на пространстве кососимметрических матриц: $[X, Y]_{B}=X B Y-Y B X$, где $B=\operatorname{diag}\left(b_{1}, b_{2}, b_{3}\right)$ - диагональная матрица. Более подробно такую структуру мы обсудим ниже.

Эта новая скобка согласована со стандартной скобкой Пуассона-Ли на $s o(3)\{$,$\} и$ со скобкой с "замороженным аргументом" $\{,\}_{g}$, где $g \in s o(3)$ - произвольный элемент. Заметим, что согласованность скобок $\{,\}_{B}$ и $\{,\}_{g}$ имеет место только в размерности три; в случае алгебры Ли $s o(n)$ это не так.

Итак, мы имеем на пространстве so(3) три попарно согласованные скобки:

$$
\left\{M_{i}, M_{j}\right\}=-\varepsilon_{i j k} M_{k}, \quad\left\{M_{i}, M_{j}\right\}_{g}=-\varepsilon_{i j k} g_{k}, \quad\left\{M_{i}, M_{j}\right\}_{B}=-\varepsilon_{i j k} b_{k} M_{k} .
$$

Рассмотрим семейство скобок вида $\{,\}_{s}=s\{\}+,\left(\{,\}_{B}-\{,\}_{g}\right), s \in \mathbb{R}$. Взяв теперь в качестве гамильтониана функцию Казимира скобки $\{,\}_{B}-\{,\}_{g}$

$$
H=\frac{1}{2}(B M, M)-(g, M)=\frac{1}{2} \sum_{i=1}^{3} b_{i} M_{i}^{3}-\sum_{i=1}^{3} g_{i} M_{i},
$$

мы получаем на алгебре Ли $s o(n)$ систему, которая является гамильтоновой относительно каждой скобки из рассматриваемого семейства:

$$
\dot{M}_{i}=\left\{M_{i}, d H(M)\right\} .
$$

Действительно, ее можно переписать в виде $\dot{M}_{i}=\left\{M_{i}, d H_{s}(M)\right\}_{s}$, где гамильтониан имеет простой вид $H_{s}(M)=\frac{1}{s} H(M)$.

С другой стороны, система (6) является классической системой Жуковского-Вольтерра, описывающей инерционное движение уравновешенного гиростата [15], [21].

Поскольку каждая из скобок $\{,\}_{s}$ при условии $s+b_{i}>0$ изоморфна стандартной скобке Пуассона-Ли на $s o(3)$, согласно общей конструкции (теорема 2) мы можем явно указать для системы Жуковского-Вольтерра представление Лакса-Гейзенберга со спектральным параметром. Поскольку изоморфизм между скобками $\{,\}_{s}$ и $\{$,$\} зада-$ ется формулой

$$
M \rightarrow(B+s E)^{-1 / 2}(M-\widetilde{g})(B+s E)^{-1 / 2},
$$

где

$$
M=\left(\begin{array}{ccc}
0 & M_{3} & -M_{2} \\
-M_{3} & 0 & M_{1} \\
M_{2} & -M_{1} & 0
\end{array}\right), \quad \widetilde{g}=\left(\begin{array}{ccc}
0 & g_{3} /\left(b_{3}+s\right) & -g_{2} /\left(b_{2}+s\right) \\
-g_{3} /\left(b_{3}+s\right) & 0 & g_{1} /\left(b_{1}+s\right) \\
g_{2} /\left(b_{2}+s\right) & -g_{1} /\left(b_{1}+s\right) & 0
\end{array}\right),
$$

соответствующая $L$ - $A$-пара имеет вид (см. теорему 2 )

$$
L(s)=(B+s E)^{-1 / 2}(M-\widetilde{g})(B+s E)^{-1 / 2}, \quad A(s)=(B+s E)^{1 / 2} d H(M)(B+s E)^{1 / 2} .
$$

Здесь

$$
d H(M)=\left(\begin{array}{ccc}
0 & b_{3} M_{3}-g_{3} & -b_{2} M_{2}+g_{2} \\
-b_{3} M_{3}+g_{3} & 0 & b_{1} M_{1}-g_{1} \\
b_{2} M_{2}-g_{2} & -b_{1} M_{1}+g_{1} & 0
\end{array}\right)
$$

Несколько другие, но эквивалентные, представления Лакса-Гейзенберга были даны в [22] и [23]. 
5. Согласованные скобки, связанные с симметрическими парами. Случай Ковалевской и твердое тело в квадратичном потенциале. Рассмотрим полупростую алгебру Ли $G$ и ее картановское разложение $G=H+V$, где $H$-подалгебра, a $V$ - подпространство, удовлетворяющие соотношениям

$$
[H, H] \subset H, \quad[H, V] \subset V, \quad[V, V] \subset H .
$$

Это эквивалентно тому, что $G$ допускает инволютивный диффеоморфизм $\theta: G \rightarrow G$, для которого $\left.\theta\right|_{H}=\mathrm{id},\left.\theta\right|_{V}=-\mathrm{id}$ (инволюция Картана). Пара $(G, H)$ называется при этом симметрической парой.

Двойственное пространство $G^{*}$ может быть представлено в виде $G^{*}=H^{*}+V^{*}$ так, что $H^{*} \perp V, V^{*} \perp H$.

Рассмотрим еще одну алгебру Ли $G_{\theta}$, которая совпадает с $G$ как линейное пространство, а коммутатор отличается только тем, что подпространство $V$ коммутативно (коммутативный идеал): $[V, V]=0$. Для $[H, H]$ и $[H, V]$ коммутатор остается прежним. На двойственном пространстве $G^{*}$ возникают, следовательно, две различные скобки Пуассона-Ли $\{$,$\} и \{,\}_{\theta}$, отвечающие алгебрам $G$ и $G_{\theta}$ соответственно. Нетрудно видеть, что эти скобки согласованы между собой.

Кроме них на $G^{*}$ имеется еще одна скобка (с замороженным аргументом) $\{,\}_{a}$, описанная выше. Если $a \in V^{*}$, то в формуле (3) можно рассматривать любой из коммутаторов $[$,$] и [,]_{\theta}$. Они будут давать один и тот же результат.

ПРЕДЛОЖЕНИЕ 7. Если $a \in V^{*}$, по скобки $\{\},,\{,\}_{\theta} u\{,\}_{a}$ образуют семейство попарно согласованных скобок Пуассона.

Как обычно, мы предполагаем, что $G$ и $G^{*}$ отождествлены при помощи ad-инвариантной формы Киллинга. Рассмотрим линейную комбинацию $\{,\}_{\alpha \beta \gamma}=\alpha\{\}+$, $\beta\{,\}_{\theta}+\gamma\{,\}_{a}$. Прямым вычислением можно проверить, что гамильтонова относительно $\{,\}_{\alpha \beta \gamma}$ система с гамильтонианом $f$ записьвается в следующем явном виде:

$$
\dot{h}=(\alpha+\beta)([\xi, h]+[\eta, v])+\gamma[\eta, a], \quad \dot{v}=(\alpha+\beta)[\xi, v]+\alpha[\eta, h]+\gamma[\xi, a],
$$

где $d f(h+v)=\xi+\eta, h, \xi \in H, v, \eta \in V$.

ПРЕДЛОЖЕНИЕ 8. Если $\alpha+\beta \neq 0$, то скобка Пуассона $\{,\}_{\alpha \beta \gamma}$ әквивалентна (сводится линейной заменой $\kappa$ ) полупростой скобке $\{\}, u$, следовательно, любая система, являющаяся гамильтоновой относительно $\{,\}_{\alpha \beta \gamma}$, допускает естественной представление Лакса-Гейзенберга.

В качестве доказательства укажем явно соответствующую $L$ - $A$-пару. А именно, уравнения (8) могут быть переписаны в следующем виде:

$$
\dot{L}=[L, A]
$$

где

$$
L=\sqrt{\frac{\alpha}{\alpha+\beta}} h+v+\frac{\gamma}{\alpha+\beta} a, \quad A=-(\alpha+\beta)\left(\xi+\sqrt{\frac{\alpha}{\alpha+\beta}} \eta\right) .
$$

Рассмотрим в качестве следствия частньй случай $\gamma=\alpha, \beta=1$ и соответствующее ему семейство скобок $\{,\}_{\theta}+\alpha\left(\{\}+,\{,\}_{a}\right)$. В силу теоремы 2 и предложения 8 любая 
бигамильтонова относительно этого семейства система допускает представление Лакса-Гейзенберга с рациональным спектральным параметром, где

$$
L=\lambda h+v+\lambda^{2} a, \quad A=(\alpha+1)(\xi+\lambda \eta) .
$$

При этом $\lambda=\sqrt{\alpha /(\alpha+1)}$, a $d f=\xi+\eta$-дифференциал гамильтониана данной системы относительно скобки $\{,\}_{\theta}+\alpha\left(\{\}+,\{,\}_{a}\right)$. В этом случае также необходимо, чтобы $\alpha \neq-1$.

В качестве гамильтониана бигамильтоновой системы можно в данном случае рассмотреть функцию Казимира $f$ особой скобки $\{,\}_{\theta}-\left(\{\}+,\{,\}_{a}\right)$ (ее выделенность в семействе заключается в том, что она не является полупростой). В описанном случае всегда существует квадратичная (неоднородная) функция Казимира вида

$$
f(h, v)=\frac{1}{2}(C(h), h)+(v, b),
$$

где $($, ) - форма Киллинга, $C: H \rightarrow H$ - самосопряженный оператор, $b \in V$, причем $C$ и $b$ удовлетворяют соотношениям $[a, b]=0,[b, h]+[C(h), a]=0$ (это в точности эквивалентно тому, что $f$ - функция Казимира).

ПРЕДЛОЖЕНИЕ 9. Рассмотрим гамильтонову относительно скобки $\{,\}_{\theta}$ систему на $G_{\theta}^{*}$ с гамильтонианом (9):

$$
\dot{h}=[C(h), h]+[b, v], \quad \dot{v}=[C(h), v] .
$$

Эта система является бигамильтоновой относительно семейства скобок $\{,\}_{\theta}+$ $\alpha\left(\{\}+,\{,\}_{a}\right)(\alpha \neq-1)$, следовательно, допускает представление Лакса-Гейзенберга со спектральным параметром. Это представление имеет вид

$$
\dot{L}=[L, A], \quad \text { əдe } \quad L=\lambda h+v+\lambda^{2} a, \quad A=-C(h)-\lambda b .
$$

В качестве примера изложенной конструкции рассмотрим систему Бруна, описывающую движение твердого тела в квадратичном потенциале, интегрируемость которой была установлена О.И. Богоявленским [24]:

$$
\left\{\begin{array}{l}
\dot{M}=[M, \omega]-[u, I] \\
\dot{u}=[u, \omega]
\end{array}\right.
$$

В этом случае $G=g l(3), H=s o(3), V$ - пространство симметрических матриц размеpa $3 \times 3, M \in s o(3)$ - матрица кинетического момента, $u \in V$ - матрица, коэффициенты которой квадратичны относительно направляющих косинусов, $\omega=\omega(M) \in s o(3)-$ угловая скорость твердого тела, при этом $\omega_{i}=a_{i} M_{i}, a_{i}$ - величины, обратные главным моментам инерции, $I=\operatorname{diag}\left(I_{1}, I_{2}, I_{3}\right)$ - матрица инерции твердого тела.

Гамильтониан системы (в смысле скобки $\left.\{,\}_{\theta}\right)$ имеет вид $H=\frac{1}{2}(A M, M)-\operatorname{Tr} u I$. Эта функция является, как несложно заметить, функцией Казимира скобки $\{,\}_{\theta}-$ $\{\}-,\{,\}_{B}$, где $B-$ симметрическая матрица вида $B=\operatorname{diag}\left(1 /\left(a_{2} a_{3}\right), 1 /\left(a_{3} a_{1}\right)\right.$, $\left.1 /\left(a_{1} a_{2}\right)\right)$. Согласно общей конструкции эта система является бигамильтоновой относительно семейства скобок $\{,\}_{\theta}+\alpha\left(\{\}+,\{,\}_{B}\right)$ (где $\alpha \neq-1$ ) и потому может быть записана в виде пары Лакса со спектральным параметром:

$$
\frac{d}{d t}\left(\lambda M+u+\lambda^{2} B\right)=\left[\lambda M+u+\lambda^{2} B, \omega-\lambda I\right] .
$$


Аналогичная конструкция для пары $G=s o(3,2), H=s o(3) \oplus s o(2)$ приводит к $L$ - $A$-паре для волчка Ковалевской, обнаруженной Рейманом и Семеновьп-Тян-Шанским, и его обобщениям [25], [26].

Получим ее с помощью изложенного метода. В качестве алгебры $G$ рассмотрим алгебру $s o(3,2)$ матрищ размера $5 \times 5$ таких, что $X^{\top}=-J_{3,2} X J_{3,2}$, где

$$
J=\operatorname{diag}(1,1,1,-1,-1), \quad X=\left(\begin{array}{cc}
\pi_{1} & S \\
S^{\top} & \pi_{2}
\end{array}\right), \quad \pi_{1} \in s o(3), \quad \pi_{2} \in s o(2),
$$

$S$ - матрища размера $3 \times 2$. В разложении Картана подалгебра $H$ является прямой суммой $s o(3) \times s o(2), V$ состоит из матриц вида

$$
\left(\begin{array}{cc}
0 & S \\
S^{\top} & 0
\end{array}\right)
$$

В переменных $M, \alpha, \beta$ уравнения движения обобщенного волчка Ковалевской в двух однородньгх полях задаются гамильтонианом

$$
H=\frac{1}{2}\left(M_{1}^{2}+M_{2}^{2}+2 M_{3}^{2}\right)-\alpha_{1}-\beta_{2}
$$

и скобкой Пуассона, определяемой алгеброй $s o(3) \oplus \mathbb{R}^{6}$ (перед компонентами $\alpha, \beta$ в $(10)$ произвольных констант $x, y$ можно не писать в силу инвариантности структуры этой алгебры по отношению к преобразованиям подобия $\alpha \rightarrow x \alpha, \beta \rightarrow y \beta$, изменяющим только орбиту).

Элементы $h, v$ и сдвиг аргумента $I$ задаются матрицами

$$
\begin{aligned}
& h=\left(\begin{array}{cccccc}
0 & M_{3} & -M_{2} & & \\
-M_{3} & 0 & M_{1} & 0 & \\
M_{2} & -M_{1} & 0 & & \\
& 0 & & 0 & M_{4} \\
& & & -M_{4} & 0
\end{array}\right) \\
& v=\left(\begin{array}{ccccc} 
& & & \alpha_{1} & \beta_{1} \\
& 0 & & \alpha_{2} & \beta_{2} \\
& & & \alpha_{3} & \beta_{3} \\
\alpha_{1} & \alpha_{2} & \alpha_{3} & 0 \\
\beta_{1} & \beta_{2} & \beta_{3} & 0
\end{array}\right), \quad I=\left(\begin{array}{ccccc} 
& & & 1 & 0 \\
& 0 & & 0 & 1 \\
& & & 0 & 0 \\
1 & 0 & 0 & \\
0 & 1 & 0 & 0
\end{array}\right) \text {, }
\end{aligned}
$$

при этом

$$
L=\lambda h+v+\lambda^{2} I
$$

Рассмотрим гамильтонову систему относительно скобки $\{$,$\} , гамильтониан которой$ задается квадратичной функцией Казимира скобки $\{,\}_{\theta}-\{\}-,\{,\}_{I}$ :

$$
H=\frac{1}{2}\left(M_{1}^{2}+M_{2}^{2}+M_{3}^{2}+M_{4}^{2}\right)-\alpha_{1}-\beta_{2} .
$$

Эта система соответствует шаровому волчку на алгебре $s o(3,2)$ в силовом поле. Она интегрируема, и ее матрица $A$, порождаемая $d H$, имеет вид

$$
A=\omega_{0}-\lambda I, \quad \text { где } \quad \omega_{0}=\left(\begin{array}{ccccc}
0 & -M_{3} & M_{2} & & \\
M_{3} & 0 & -M_{1} & 0 \\
-M_{2} & M_{1} & 0 & & \\
& 0 & & 0 & -M_{4} \\
& & & M_{4} & 0
\end{array}\right) \text {. }
$$


Несложно показать, что уравнения движения допускают линейный интеграл

$$
M_{3}+M_{4}=c=\text { const }
$$

Выполним с помошью него редукцию, несколько отличную от обычной редукции по моменту. Обозначим интегралы, коммутирующие с гамильтонианом (12) (в структуре $\left.\{,\}_{\theta}\right)$, через

$$
F_{i}\left(M, \alpha, \beta, M_{4}\right), \quad i=1,2,3,
$$

и выберем новые переменные $M, \alpha, \beta, y=c M_{3}+M_{4}$, которые удовлетворяют следующим свойствам:

1) $M, \alpha, \beta$ образуют замкнутую подалгебру относительно скобки $\{,\}_{\theta}$;

2) $y$ коммутирует (относительно $\{,\}_{\theta}$ ) с гамильтонианом (12) и интегралами $F_{i}$, $i=1,2,3$.

Справедливо следующее

ПРеДЛОЖЕНИЕ 10. Система с гамильтонианом $\bar{H}(M, \alpha, \beta)=\left.H(M, \alpha, \beta, y)\right|_{y=c}$, где $c=$ const, имеет инволютивные первые интегралы вида

$$
\bar{F}_{i}(M, \alpha, \beta)=\left.F_{i}(M, \alpha, \beta, y)\right|_{y=c} .
$$

ДокаЗАТЕльСтво - прямая проверка равенств $\left\{\bar{F}_{i}, \bar{H}\right\}_{\theta}=0$ и $\left\{\bar{F}_{i}, \bar{F}_{j}\right\}_{\theta}=0$. Таким образом, редуцированная система также интегрируема, и ее гамильтониан имеет вид

$$
\bar{H}=\frac{1}{2}\left(M_{1}^{2}+M_{2}^{2}+2 M_{3}^{2}\right)-c M_{3}-\alpha_{1}-\beta_{2} .
$$

Матрица $A$, соответствующая $d \bar{H}$, имеет вид

$$
A=\omega-\lambda I, \quad \text { где } \quad \omega=\left(\begin{array}{ccccc}
0 & -2 M_{3}+c & M_{2} & & \\
2 M_{3}-c & 0 & -M_{1} & 0 \\
-M_{2} & M_{1} & 0 & & \\
& 0 & & 0 & 0 \\
& & & 0 & 0
\end{array}\right) .
$$

В матрице $L$ при этом необходимо положить $M_{4}=c-M_{3}$. Полный набор первых интегралов может быть получен при разложении $\operatorname{Tr} L^{k}$ по спектральному параметру.

Отметим, что описанная процедура редукции, проведенная для алгебры $(s o(3) \oplus s o(2))$ $\oplus_{S} \mathbb{R}^{6}$, входящей в пучок, неможет быть проведена одновременно для всех скобок пучка и соответственно не может индуцировать (редуцированную) бигамильтонову структуру. По-видимому, волчок Ковалевской (в отличие от интегрируемых систем, рассматриваемых ранее) вообще не допускает бигамильтонова описания.

Отметим, что аналогичная конструкция для алгебры Ли $G=s u(2,1)$ позволяет построить представление Лакса со спектральным параметром для волчка Горячева-Чаплыгина.

6. Лиевы пучки и многомерные случаи Эйлера и Клебша. Перейдем теперь к другому сюжету из теории (конечномерных) алгебр Ли. 
ОПРЕДЕЛЕНИЕ 6. Лиевым пучком на конечномерном пространстве $L$ назьвается линейное семейство лиевых структур. Это означает, что на $L$ задано семейство коммутаторов $[,]_{C}$, каждьй из которых задает на $L$ структуру алгебры Ли, причем параметр $C$ пробегает некоторое линейное пространство $V$ и, кроме того, вьполняется следующее естественное условие линейности:

$$
[,]_{\lambda A+\mu B}=\lambda[,]_{A}+\mu[,]_{B} \quad \text { для } \quad A, B \in V, \quad \lambda, \mu \in V .
$$

Это определение совершенно аналогично понятию согласованных пуассоновьх структур. Действительно, повторяя определение 3, две лиевы структуры естественно называть согласованными, если любая их линейная комбинация снова задает на $L$ структуру алгебры Ли (т.е. удовлетворяет тождеству Якоби). Это в точности эквивалентно тому, что структуры порождают двумерный лиев пучок. Обратно, любой лиев пучок состоит из попарно согласованных лиевых структур.

Связь между согласованными скобками Пуассона и лиевьми пучками очень проста. Если на линейном пространстве $L$ задан лиев пучок $[,]_{C}, C \in V$, то на двойственном пространстве $L^{*}$ возникает семейство попарно согласованньх скобок Пуассона-Ли $\{,\}_{C}, C \in V$.

В качестве примера рассмотрим пространство кососимметрических матриц $L$, отождествленное с алгеброй Ли $s o(n)$. Вводя естественное инвариантное скалярное произведение $(X, Y)=-\operatorname{Tr} X Y$, мы отождествляем $L$ с $L^{*}$. Далее рассмотрим на $L$ семейство алгебр Ли, коммутаторы которых задаются так:

$$
[X, Y]_{C}=X C Y-Y C X
$$

где $C$ - произвольная симметричная матрица. На двойственном пространстве $L^{*}=L$ эти алгебры порождают семейство скобок Ли-Пуассона $\{\}$,$C . Гамильтоновость сис-$ темы $v$ относительно скобки $\{\}$,$C означает, что$

$$
v(X)=X d H(X) C-C d H(X) X
$$

для некоторой гладкой функции $H(X): L \rightarrow \mathbb{R}$.

Отметим, что

1) все эти скобки согласованы между собой,

2) скобка $\{,\}_{C}$ полупроста тогда и только тогда, когда матрица $C$ невырождена.

Из второго свойства, в частности, следует, что в случае невырожденной матрицы $C$ выписанное выше уравнение может быть записано в лаксовой форме (т.е. в виде обычного коммутатора). Для этого нужно сделать следующую замену:

$$
X \rightarrow C^{1 / 2} L C^{1 / 2}, \quad d H(X) \rightarrow C^{-1 / 2} A C^{-1 / 2} .
$$

Подставляя, мы получим $C^{1 / 2} \dot{L} C^{1 / 2}=C^{1 / 2}(L A-A L) C^{1 / 2}$ или, что то же самое, $\dot{L}=[L, A]$.

Рассмотрим теперь уравнения Эйлера движения многомерного твердого тела:

$$
\dot{X}=X \Omega-\Omega X
$$


где $X$ и $\Omega$ - кососимметрические матрицы, связанные соотношением $X=B \Omega+\Omega B$, и $B$ - диагональная невырож денная матрица.

Одно из представлений Лакса со спектральньм параметром в этом случае хорошо известно [18]. Здесь мы опишем еще одно представление [2], связанное с описанным вьше семейством скобок. В [1] показано, что уравнения (14) являются гамильтоновыми относительно семейства скобок $\{,\}_{B^{2}+\lambda E}$. Используя это обстоятельство и тот факт, что эта скобка полупроста почти для всех $\lambda$, мы можем переписать уравнения для каждой алгебры Ли $[,]_{B^{2}+\lambda E}$ в лаксовой форме. Приведем конечный результат.

ПРЕДЛОЖЕНИЕ 11. Система уравнений (14) может быть записана в следующем әквивалентном виде:

$$
\frac{d L(\lambda)}{d t}=[L(\lambda), A(\lambda)]
$$

$2 \partial e$

$$
\begin{aligned}
& L(\lambda)=\left(B^{2}+\lambda E\right)^{-1 / 2} X\left(B^{2}+\lambda E\right)^{-1 / 2} \\
& A(\lambda)=\left(B^{2}+\lambda E\right)^{-1 / 2}(\lambda \Omega-B \Omega B)\left(B^{2}+\lambda E\right)^{-1 / 2} .
\end{aligned}
$$

Эквивалентность этого представления системе (14) легко проверяется прямым вычислением. Здесь, впрочем, интересна его связь с семейством скобок. Прокомментируем ее. Поскольку система (14) гамильтонова относительно скобки $\{,\}_{B^{2}+\lambda E}$, мы можем представить $\dot{X}$ в виде

$$
\dot{X}=X d H_{\lambda}(X)\left(B^{2}+\lambda E\right)-\left(B^{2}+\lambda E\right) d H_{\lambda}(X) X
$$

Несложно проверить, что здесь

$$
d H_{\lambda}(X)=\left(B^{2}+\lambda E\right)^{-1}(\lambda \Omega-B \Omega B)\left(B^{2}+\lambda E\right)^{-1} .
$$

Чтобы теперь из этого выражения получить представление с обычным коммутатором, нужно сделать замену, которая уже была указана вьше:

$X=\left(B^{2}+\lambda E\right)^{1 / 2} L(\lambda)\left(B^{2}+\lambda E\right)^{1 / 2}, \quad d H_{\lambda}(X)=\left(B^{2}+\lambda E\right)^{-1 / 2} A(\lambda)\left(B^{2}+\lambda E\right)^{-1 / 2}$,

что сразу приводит нас к доказьваемому результату.

ЗАмЕчАниЕ. Отметим, что если диагональные элементы $b_{i}$ матрицы $B$ различны, то при $\lambda=-\min b_{i}^{2}$ алгебра Ли $[,]_{B^{2}+\lambda E}$ изоморфна алгебре Ли $e(n-1)=s o(n-1)+$ $\mathbb{R}^{n-1}$ групшы движений $(n-1)$-мерного (аффинного) пространства. Тем самым мы видим, что уравнения движения $n$-мерного твердого тела (14) при помощи линейной замены переводятся в некоторою интегрируемую систему на алгебре $e(n-1)$. Оказывается, эта система совпадает с $(n-1)$-мерным аналогом случая Клебша движения твердого тела в идеальной жидкости, который был обнаружен А. М. Переломовым [25]. Для $n=4$ этот неожиданньй изоморфизм между двумя интегрируемыми задачами был установлен А. И. Бобенко [27], обший случай обсуждается в [2]. 
7. Еще один экзотический пучок, связанный со случаями Стеклова и Ляпунова. Здесь мы обсудим еще один пример лиева пучка, которьй был обнаружен Ю.Н. Федоровым [28] на основе анализа классической работы Кеттера [29].

Рассмотрим прямую сумму пространств кососимметрических матриц $L=s o(n)+$ $s o(n)$. Элементы этого пространства будем записывать в виде пары $(X, Y), X \in s o(n)$, $Y \in s o(n)$. Пара коммутаторов, порождающих пучок, имеет вид

$$
\begin{aligned}
& {\left[\left(X_{1}, Y_{1}\right),\left(X_{2}, Y_{2}\right)\right]_{0}=\left(\left[X_{1}, X_{2}\right],\left[X_{1}, Y_{2}\right]+\left[Y_{1}, X_{2}\right]-\left[X_{1}, X_{2}\right]_{B}\right)} \\
& {\left[\left(X_{1}, Y_{1}\right),\left(X_{2}, Y_{2}\right)\right]_{1}=\left(\left[X_{1}, X_{2}\right]_{B},\left[Y_{1}, Y_{2}\right]\right) .}
\end{aligned}
$$

Здесь через $[,]_{B}$ обозначен коммутатор вида $\left[X_{1}, X_{2}\right]_{B}=X_{1} B X_{2}-X_{2} B X_{1}$, где $B$ симметричная матрица. В нашем случае мы считаем ее диагональной.

Несложно проверить, что данные коммутаторы согласованы, т.е. любая их линейная комбинация удовлетворяет тождеству Якоби и, следовательно, задает структуру алгебры Ли на пространстве $s o(n)+s o(n)$.

Посмотрим, чему изоморфны алгебры Ли из рассматриваемого пучка $[,]_{0+\lambda \cdot 1}=$ $[,]_{0}+\lambda[,]_{1}$.

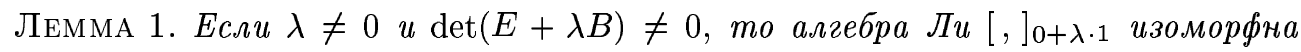
$s o(n)+s o(n)$ со стандартным матричным коммутатором. При этом изоморфизм ч задается следуюшими явными формулами:

$$
\varphi(X, Y)=\left((E+\lambda B)^{1 / 2} X(E+\lambda B)^{1 / 2}, \lambda Y+X\right) .
$$

\section{ДокАЗАТЕЛЬСТво - явная проверка.}

Из этого утверждения легко вытекает вид инвариантов коприсоединенного представления на $L^{*}$. Как обычно, мы отождествляем $L=s o(n)+s o(n)$ и $L^{*}=(s o(n)+s o(n))^{*}$ при помоши скалярного произведения $\langle(X, Y),(Z, P)\rangle=\operatorname{Tr}(X Z+Y P)$. Операторы $\varphi^{*}: L^{*} \rightarrow L^{*}$ и $\varphi^{*-1}: L^{*} \rightarrow L^{*}$ имеют тогда следующий вид:

$$
\begin{aligned}
\varphi^{*}(Z, P) & =\left((E+\lambda B)^{1 / 2} Z(E+\lambda B)^{1 / 2}+P, \lambda P\right), \\
\varphi^{*-1}(Z, P) & =\left((E+\lambda B)^{-1 / 2}\left(Z-\lambda^{-1} P(E+\lambda B)^{-1 / 2}\right), \lambda^{-1} P\right) .
\end{aligned}
$$

Инварианты прямой суммы $L=s o(n)+s o(n)$ при стандартном представлении хорошо известны. Это функции вида

$$
\operatorname{Tr} Z^{2 k}, \quad \operatorname{Tr} P^{2 k}
$$

Используя (14) и явный вид оператора $\varphi^{*-1}$, получаем явный вид функций Казимира скобки $\{,\}_{0+\lambda \cdot 1}$ :

$$
\operatorname{Tr}\left(\left(Z-\lambda^{-1} P\right)(E+\lambda B)^{-1}\right)^{2 k}, \quad \operatorname{Tr} P^{2 k} .
$$

При $\lambda=0$ эта формула не очень хорошо работает. Чтобы получить хорошую асимптотику в нуле, нужно вместо первого инварианта рассмотреть следующий:

$$
\begin{aligned}
& \frac{1}{\lambda}\left(\operatorname{Tr}\left((\lambda Z-P)(E+\lambda B)^{-1}\right)^{2 k}-\operatorname{Tr} P^{2 k}\right) \\
& \quad=\frac{1}{\lambda}\left(\operatorname{Tr}\left((\lambda Z-P)\left(E-\lambda B+\lambda^{2} B^{2}+\lambda^{3} B^{3}-\cdots\right)\right)^{2 k}-\operatorname{Tr} P^{2 k}\right) .
\end{aligned}
$$


Легко проверяется, что полученное выражение является степенным рядом по $\lambda$, причем первьй (свободный) член этого ряда имеет вид

$$
\operatorname{Tr}(Z+P B) P^{2 k-1} .
$$

Ясно, что это функция Казимира скобки $\{,\}_{0}$. Вместе с функциями вида $\operatorname{Tr} P^{2 k}$ они образуют полньй набор.

Какова структура алгебры Ли $[,]_{0}$ ? Оказывается, она изоморфна полупрямой сумме алгебры $s o(n)$ и коммутативного идеала $\mathbb{R}^{[n(n-1) / 2]}$ по присоединенному представлению. Стандартный коммутатор для этой полупрямой суммы задается на пространстве $L$ естественным способом:

$$
\left[\left(X_{1}, Y_{1}\right),\left(X_{2}, Y_{2}\right)\right]^{\sim}=\left(\left[X_{1}, X_{2}\right],\left[X_{1}, Y_{2}\right]+\left[Y_{1}, X_{2}\right]\right)
$$

Изоморфизм между этим стандартным коммутатором и “деформированньм” [, ]0 задается отображением

$$
\psi(X, Y)=\left(X, Y-\frac{1}{2}(B X+X B)\right) .
$$

Напомним, что это означает следующее:

$$
\psi\left[\left(X_{1}, Y_{1}\right),\left(X_{2}, Y_{2}\right)\right]_{0}=\left[\psi\left(X_{1}, Y_{1}\right), \psi\left(X_{2}, Y_{2}\right)\right] .
$$

Сопряженньй оператор имеет вид

$$
\psi^{*}(Z, P)=\left(Z-\frac{1}{2}(B P+P B), P\right) .
$$

Таким образом, уравнения Эйлера в смысле скобки $\{,\}_{0}$ приводятся к стандартным уравнениям в смысле скобки, отвечающей полупрямой сумме $s o(n)+{ }_{\mathrm{ad}} R^{[n(n-1) / 2]}$, при помощи замены вида $(Z, P) \rightarrow(M, P)$,

$$
Z=M-\frac{1}{2}(B P+P B), \quad P=P .
$$

Опишем теперь семейство гамильтонианов, порождающих системы, являющиеся гамильтоновыми относительно каждой скобки из нашего семейства. Легко видеть, что такому свойству удовлетворяют функции Казимира скобок $\{,\}_{0}+\lambda\{,\}_{1}$ максимального ранга. Поскольку нас интересуют только квадратичные гамильтонианы, то мы можем рассмотреть семейство функций, являющихся линейньми комбинациями описанных выше квадратичных функций Казимира. Можно проверить, что функции из этого семейства имеют следующий общий вид:

$$
\begin{aligned}
H(Z, P)= & \sum_{i<j} \frac{c_{i}-c_{j}}{b_{i}-b_{j}} Z_{i j}^{2}+2 \sum_{i<j} \frac{b_{i} c_{i}-b_{j} c_{j}}{b_{i}-b_{j}} Z_{i j} P_{i j} \\
& +\sum_{i<j} \frac{b_{i}^{2} c_{i}-b_{j}^{2} c_{j}}{b_{i}-b_{j}} P_{i j}^{2}+\text { const } \sum_{i<j} P_{i j}^{2} .
\end{aligned}
$$

Последнее слагаемоев этой сумме на самом деле большого смысла не имеет, поскольку является функцией Казимира для каждой из скобок. 
ПРЕДЛОЖЕНИЕ 12. Пусть гамильтониан $Н$ имеет вид (15). Тогда он порохдает бигамильтонову систему. А именно, существует функиия $\widetilde{H}$ такая, что справедливо тохсдество

$$
\operatorname{sgrad}_{1} H=\operatorname{sgrad}_{0} \widetilde{H} .
$$

При әтом гамильтониан $\widetilde{H}$ может быть взят в виде

$$
\widetilde{H}(Z, P)=\sum_{i<j} \frac{b_{i} c_{i}-b_{j} c_{j}}{b_{i}-b_{j}} Z_{i j}^{2}+2 \sum_{i<j} \frac{b_{i}^{2} c_{i}-b_{j}^{2} c_{j}}{b_{i}-b_{j}} Z_{i j} P_{i j}+\sum_{i<j} \frac{b_{i}^{3} c_{i}-b_{j}^{3} c_{j}}{b_{i}-b_{j}} P_{i j}^{2} .
$$

Отметим, что гамильтониан $\widetilde{H}$ определен неоднозначно. $\mathrm{K}$ нему всегда можно добавить произвольную функцию Казимира скобки $\{,\}_{0}$.

Напомним, что равенство (16) может быть интерпретировано как изоморфизм между системой на полупрямой сумме $s o(n)+{ }_{\mathrm{ad}} \mathbb{R}^{[n(n-1) / 2]}$ и системой на прямой сумме $s o(n) \oplus s o(n)$. Однако здесь обе скобки имеют не совсем стандартньй вид; чтобы привести их к стандартной форме, нужно произвести некоторые замены (они были уже описаны вьше). В итоге мы получим следующий результат.

ПРЕДЛОЖЕНИЕ 13. Рассмотрим на пространстве $G=s o(n) \oplus \operatorname{so}(n)$ со стандартной скобкой гамильтониан следующего вида:

$$
H_{G}(X, Y)=\sum_{i<j} \frac{c_{i}-c_{j}}{b_{i}-b_{j}} b_{i} b_{j} X_{i j}^{2}+2 \sum_{i<j} \frac{b_{i} c_{i}-b_{j} c_{j}}{b_{i}-b_{j}} \sqrt{b_{i} b_{j}} X_{i j} Y_{i j}+\sum_{i<j} \frac{b_{i}^{2} c_{i}-b_{j}^{2} c_{j}}{b_{i}-b_{j}} Y_{i j}^{2} .
$$

Рассмотрим на пространстве $F=\left(\operatorname{so}(n)+{ }_{\text {ad }} \mathbb{R}^{[n(n-1) / 2]}\right)^{*}$ со стандартной скобкой Пуассона гамильтониан вида

$$
\begin{aligned}
H_{F}(M, P)= & \sum_{i<j} \frac{b_{i} c_{i}-b_{j} c_{j}}{b_{i}-b_{j}}\left(M_{i j}-\frac{1}{2}\left(b_{i}+b_{j}\right) P_{i j}\right)^{2} \\
& +2 \sum_{i<j} \frac{b_{i}^{2} c_{i}-b_{j}^{2} c_{j}}{b_{i}-b_{j}}\left(M_{i j}-\frac{1}{2}\left(b_{i}+b_{j}\right) P_{i j}\right) P_{i j}+\sum_{i<j} \frac{b_{i}^{3} c_{i}-b_{j}^{3} c_{j}}{b_{i}-b_{j}} P_{i j}^{2} .
\end{aligned}
$$

Тогда соответствующие этим гамильтонианом системы сводятся друг к другу при помощи следующей линейной замены переменных:

$$
M=B^{1 / 2} X B^{1 / 2}+\frac{1}{2}(B Y+Y B), \quad P=Y .
$$

Как теперь получить представление Лакса со спектральньм параметром для гамильтонианов из описанного семейства?

Рассмотрим бигамильтоново векторное поле

$$
v=\operatorname{sgrad}_{1} H=\operatorname{sgrad}_{0} \widetilde{H} .
$$

Оно, как мы знаем, может быть представлено как гамильтоново векторное поле относительно линейной комбинации $\{,\}_{0}+\lambda\{,\}_{1}$ :

$$
v=\operatorname{sgrad}_{0+\lambda \cdot 1} H_{0+\lambda \cdot 1} \text {. }
$$


Гамильтониан при этом имеет следующий явный вид:

$$
H_{0+\lambda \cdot 1}=\sum_{i<j} \frac{a_{i}-a_{j}}{b_{i}-b_{j}} Z_{i j}^{2}+2 \sum_{i<j} \frac{b_{i} a_{i}-b_{j} a_{j}}{b_{i}-b_{j}} Z_{i j} P_{i j}+\sum_{i<j} \frac{b_{i}^{2} a_{i}-b_{j}^{2} a_{j}}{b_{i}-b_{j}} P_{i j}^{2},
$$

где $a_{i}=c_{i} b_{i}\left(1+\lambda b_{i}\right)^{-1}$.

Теперь мы можем переписать уравнение (17) в лаксовой форме, пользуясь соображениями, описанными выше (теорема 2):

$$
\dot{L}=[L, A]
$$

В нашем случае $L=\varphi^{*-1}(Z, P)$, т.е.

$$
L=\left(\begin{array}{cc}
(E+\lambda B)^{-1 / 2}\left(Z-\lambda^{-1} P\right)(E+\lambda B)^{-1 / 2} & 0 \\
0 & \lambda^{-1} P
\end{array}\right)
$$

и

$$
A=\varphi\left(d H_{0+\lambda \cdot 1}(Z, P)\right) .
$$

Благодарности. Авторы искренне признательны И. С. Мамаеву за полезные обсуждения и замечания.

\section{СПИСОК ЦИТИРОВАННОЙ ЛИТЕРАТУРЫ}

[1] Болсинов А. В. Согласованные скобки Пуассона на алгебрах Ли и полнота семейств функций в инволюции // Изв. АН СССР. Сер. матем. 1991. Т. 55. №1. С. 68-92.

[2] Болсинов А. В. Многомерные случаи Эйлера и Клебша и лиевы пучки // Тр. семинара по векторному и тензорному анализу. Вып. 24. М.: Изд-во МГУ, 1991. С. 8-12.

[3] Гельфанд И. М., Дорфман И.Я. Гамильтоновы операторы и связанные с ними алгебраические структуры // Функцион. анализ и его прилож. 1979. Т. 13. № 4. С. 13-30.

[4] Мешерлков М. В. О характеристическом свойстве тензора инерции многомерного твердого тела // УМН. 1983. Т. 38. № 5. С. 201-202.

[5] Bogoyavlenskij O. I. Theory of tensor invariants of integrable Hamiltonian systems I. Incompatible Poisson structures // Comm. Math. Phys. 1996. V. 180. P. 529-586.

[6] Bogoyavlenskij O. I. Theory of tensor invariants of integrable Hamiltonian systems II. Theorem on symmetries and its applications // Comm. Math. Phys. 1997. V. 184. P. 301-365.

[7] Magri F. A simple model of the integrable Hamiltonian equation // J. Math. Phys. 1978. V. 19. № 5. P. 1156-1162.

[8] Panasyuk A. Veronese webs for bihamiltonian structures of higher corank // Poisson Geometry. V. 51: Warszawa, 2000. P. 251-261.

[9] Gelfand I. M., Zakharevich I. S. On the local geometry of a bihamiltonian structure // The Gelfand Mathematical Seminars 1990-1992. Boston: Birkhäuser, 1993. P. 51-112.

[10] Гельфанд И. М., Захаревич И. С. Ткани, кривые Веронезе и бигамильтоновы системы // Функцион. анализ и его прилож. 1991. Т. 99. № 1. С. 150-178.

[11] Семенов-Тян-Шанский М. А. Что такое классическая $r$-матрица // Функцион. анализ и его прилож. 1983. Т. 17. №4. С. 17-33.

[12] Olver P. Canonical forms and integrability of bi-Hamiltonian systems // Phys. Lett. A. 1990. V. 148. № 3, 4. P. 177-187.

[13] Turiel F. G. Classification locale d'un couple des formes sympléctiques Poisson-compatible // C. R. Acad. Sci. Paris. Sér. I. 1989. V. 308. P. 575-578.

[14] Brouzet R. About the existence of recursion operator for completely integrable Hamiltonian systems near a Liouville torus // J. Math. Rhys. 1993. V. 34. P. 1309-1313. 
[15] Volterra V. Sur la théorie des variations des latitudes // Acta Math. 1899. V. 22. P. 201-358.

[16] Мишенко А. С., Фоменко А.Т. Уравнения Эйлера на конечномерных группах Ли // Изв. АН СССР. Сер. матем. 1978. Т. 42. № 2. С. 386-415.

[17] Мищенко А. С., Фоменко А. Т. Интегрируемость уравнений Эйлера на полупростых алгебрах Ли // Труды семинара по векторному и тензорному анализу. Вып. 19. М.: Изд-во МГУ, 1979. С. 3-94.

[18] Манаков С. В. Замечание об интегрировании уравнений Эйлера динамики $n$-мерного твердого тела // Функцион. анализ и его прилож. 1976. Т. 10. № 4. С. 93-94.

[19] Adler M., van Moerbeke P. Geodesic flow on so(4) and intersection of quadrics // Proc. Nat. Acad. Sci. USA. 1984. V. 81. P. 4613-4616.

[20] Reyman A. G., Semenov-Tyan-Shansky M. A. A new integrable case of the motion of the 4-dimensional rigid body // Comm. Math. Phys. 1986. V. 105. P. 461-472.

[21] Ж Жуковский Н. Е. О движении твердого тела, имеющего полости, наполненные однородной капельной жидкостью I, II, III // Журнал фииз. хим. общества. 1885. Т. 17 (№отд. 1, 6; 7; 8). С. $81-113,145-199,231-280$.

[22] Федоров Ю.Н. Представления Лакса со спектральньм параметром, определенном на накрытиях суперэллиптических кривых // Матем. заметки. 1993. Т. 54. №1. С. 94-109.

[23] Борисов А.В., Мамаев И.С. Пуассоновы структуры и алгебры Ли в гамильтоновой механике. Ижевск: Издательский дом "Удмуртский университет", 1999.

[24] Богоявленский О.И.Интегрируемые уравнения Эйлера на алгебрах Ли, возникающие в задачах математической физики // Изв. АН СССР. Сер. матем. 1984. Т. 48. № 5. С. 883-938.

[25] Ольшанецкий М. А., Переломов А. Г., Рейман А. Г., Семенов-Тян-Шанский М. А. Интегрируемые системы. II // Современные проблемы математики. Фундамент. направления. Т. 16. М.: ВИНИТИ, 1987. С. 86-226.

[26] Рейман А. Г., Семенов-Тян-Шанский М.А. Лаксово представление со спектральным параметром для волчка Ковалевской и его обобщений // Функцион. анализи его прилож. 1988. T. 22. № 2. C. $87-88$.

[27] Бобенко А.И. Об интегрировании уравнений Эйлера на $e(3)$ и $s o(4)$. Изоморфизм интегрируемых случаев // Функцион. анализ и его прилож. 1986. Т. 20. № 1. С. 64-66.

[28] Болсинов А. В., Федоров Ю. Н. Многомерные интегрируемые обобщения систем Стеклова-Ляпунова // Вестн. МГУ. Сер. 1. Матем., мех. 1992. №6. С. 53-56.

[29] Kötter F. Über die von Steklow und Liapunow entdeckten integralen Falle der Bewegung eines starren Körpers in einer Flüssigkeit // Sitzungsber. Königlich preuslischen Acad. Wiss. Berlin. 1900. V. 6. P. 79-87.

[30] Bogoyavlenskij O. I. Extended integrability and bi-Hamiltonian systems // Comm. Math. Phys. 1998. V. 196. P. 19-51. 